\title{
Online calculator to predict early mortality in patient with surgically treated recurrent lower-grade glioma
}

\author{
Ruolun Wei ${ }^{1}$, Chao Zhao ${ }^{2}$, Jianguo $\mathrm{Li}^{3}$, Fengdong Yang ${ }^{1}$, Yake Xue ${ }^{1}$ and Xinting Wei ${ }^{{ }^{*}}$ (D)
}

\begin{abstract}
Purpose: The aim of this study was to investigate the epidemiological characteristics and associated risk factors of recurrent lower-grade glioma [LGG] (WHO grades II and III) according to the 2016 updated WHO classification paradigm and finally develop a model for predicting early mortality (succumb within a year after reoperation) in recurrent LGG patients.

Methods: Data were obtained from consecutive patients who underwent surgery for primary LGG and reoperation for tumor recurrence. The end point "early mortality" was defined as death within 1 year after the reoperation. Predictive factors, including basic clinical characteristics and laboratory data, were retrospectively collected.

Results: A final nomogram was generated for surgically treated recurrent LGG. Factors that increased the probability of early mortality included older age $(P=0.042)$, $\mathrm{D}$-dimer $>0.187(P=0.007), \mathrm{RDW}>13.4(P=0.048), \mathrm{PLR}>100.749$ $(P=0.014), \mathrm{NLR}>1.815(P=0.047), 1 \mathrm{p} 19 \mathrm{q}$ intact $(P=0.019)$, IDH1-R132H Mutant $(P=0.048)$, Fib $\leq 2.80(P=0.018)$, lack of Stupp concurrent chemoradiotherapy $(P=0.041)$, and an initial symptom of epilepsy $(P=0.047)$. The calibration curve between the prediction from this model and the actual observations showed good agreement.

Conclusion: A nomogram that predicts individualized probabilities of early mortality for surgically treated recurrent LGG patients could be a practical clinical tool for counseling patients regarding treatment decisions and optimizing therapeutic approaches. Free online software implementing this nomogram is provided at https://warrenwrl.shiny apps.io/RecurrenceGliomaEarlyM/
\end{abstract}

Keywords: Lower-grade glioma, Early mortality, Recurrent glioma, Nomogram

\section{Introduction}

Gliomas are among the most common adult brain tumors. Unlike cancers originating from other organs, tumors in the central nervous system (CNS) obey a unique histological and grading system [1]. Following the identification of key oncological alterations providing superior prognostication than pathological identification, the understanding of glioma and other

\footnotetext{
*Correspondence: fccweixt@zzu.edu.cn

${ }^{1}$ Department of Neurosurgery, The First Affiliated Hospital of Zhengzhou

University, Jianshe East Road, Zhengzhou, China

Full list of author information is available at the end of the article
}

central nervous system tumors has evolved and led to the updated 2016 World Health Organization (WHO) Central Nervous System Tumor Classification. The previous grading of "low-grade glioma," which referred to WHO grade II gliomas, has been renewed and reclassified as "lower-grade glioma" to encompass WHO grade II and III tumors [2]. Recurrence is typically inevitable for most lower-grade glioma (LGG) patients due to the infiltrative nature of the tumor. An LGG that has been resected in a prior surgery may recur in their initial grade or undergo malignant transformation to a higher grade, such as oligodendroglial to anaplastic-oligodendroglial 
tumors or anaplastic-astrocytoma to secondary glioblastoma (sGBM).

The treatment decision for recurrent LGG has consistently been of concern for neurosurgeons and oncologists. The primary role of reoperation in the management of recurrent low-grade gliomas has not been clarified [3], but studies have confirmed that reoperation provides a significant benefit for patients with recurrent LGG [4]. However, the rationality of reoperation could be questioned if the patient succumbs to death within one year after reoperation of recurrent LGG, especially as salvage chemotherapy may prolong patient post recurrence survival than one year. To this end, in the present study, we generated and validated a nomogram to assist clinical decision-making by distinguishing recurrent LGG patients at high risk of early mortality.

\section{Material and methods \\ Study population}

In this retrospective study, adult patients (aged $\geq 18$ years) who underwent primary surgery and histopathology leading to a diagnosis of WHO grade II or III glioma and consecutively underwent reoperation for tumor recurrence between January 2000 and June 2021 at The First Affiliated Hospital of Zhengzhou University were included. All patients included conformed to reoperative and postoperative Karnofsky Performance Status (KPS) scores $\geq 70$. Electronic medical records and, if needed, paper charts with complete data of pre- and postoperative routine tests were used. The exclusion criteria were as follows: 1) a clinical history of chronic diseases more than 1 year; 2 ) obvious systemic diseases; 3 ) patients with other malignancies; 4) no history of receiving chemotherapy or radiotherapy before the operation; and 4) perioperative surgery-related mortality. The final cohort included a total of 106 patients. The following variables were obtained from the cohort and included in the study: age at diagnosis, sex, initial symptoms, oncology markers, tumor size, tumor location and laboratory data. The study protocol was approved by the Ethics Committee of the First Affiliated Hospital of Zhengzhou University, and the requirement for signed informed consent from patients was waived due to the retrospective nature of the analysis. All patient data were treated with confidentiality, in accordance with the Declaration of Helsinki.

\section{Histological evaluation}

Histological evaluations were performed on H\&E-stained archival slides. All cases were reviewed by neuropathologists according to the latest WHO classification criteria for tumors of the CNS, and the definitive diagnosis was reaffirmed according to the 2016 WHO classification.

\section{Immunohistochemical staining and analyses}

Immunohistochemical staining was performed on an automated immunohistochemical staining system (BenchMark XT, USA. Stained tissue microarray slides were scanned with a Leica Aperio AT2 scanner at 400x magnification. Digital images were analyzed by Leica Aperio ImageScope software with the Nuclear v9 algorithm. The following biomarkers were recorded: 1) IDH1$\mathrm{R} 132 \mathrm{H}$ (isocitrate dehydrogenase 1), 2) 1p19q, 3) P53, 4) ATRX, and 5) Ki67. IDH1 and 1p19q codeletion and alpha thalassemia/mental retardation syndrome X-linked (ATRX) status were scored as positive or negative. P53 status was quantified as the percentage of stained nuclei: less than $10 \%$ of stained nuclei indicated an absence of immunoreactivity; $10-30 \%$ indicated a score of $1+; 30.1-$ $50 \%$ indicated a score of $2+$; and more than $50 \%$ indicated a score of $3+$. Scores of -1 or $1+$ were regarded as P53 negative, and 2+ and 3+ were regarded as P53 positive. The Ki-67 index was also calculated according to the percentage of Ki-67 positive tumor cells present in the sample.

\section{Laboratory test analyses}

Preoperative laboratory markers of the first operation included NLR: neutrophil-to-lymphocyte ratio, PLR: platelet-to-lymphocyte ratio, LMR: lymphocyte-tomonocyte ratio, RDW: red blood cell distribution width, FIB: fibrinogen level, and DD: D-dimer level.

\section{Follow-up}

Patients were followed by clinical and radiological exams periodically. Tumor recurrence or progression was defined using Response Assessment in Neuro-Oncology (RANO) criteria with both clinical status deterioration and radiologic changes on MRI.

Overall survival (OS) was defined as the interval from the date of initial diagnosis (data of first surgery) to the date of death. Time to recurrence (TTR) was defined as the interval between the date of initial diagnosis (data of first surgery) and the progression of disease. Post recurrence survival (PRS) was defined as the survival after recurrence and reoperation. The interval was censored at the last follow-up visit. The patients' vital status and date of last follow-up were last updated on May 21, 2021.

Survival analyses were performed using the KaplanMeier method. Survival distributions were compared using log-rank tests.

\section{Statistical method}

For continuous variables, independent sample $t$ tests were used to compare distributions. For categorical variables, the chi-squared test or Fisher's exact test was used 
as appropriate. The optimal cutoff value for each hematological marker was determined by the best area under the curve (AUC) in receiver operating characteristic (ROC) curve analysis. Patients were classified binarily according to the cutoff values. To filter out early mortality-associated factors from all covariates, univariable Cox analysis was performed, and all significant variables from the analysis were included in multivariate Cox analysis. The hazard ratio and $P$ value from the univariate and multivariate Cox analyses are demonstrated in a forest plot. The Kaplan-Meier method was used to generate survival curves, and the log-rank test was used for comparison. Based on the multivariate Cox analysis, a model for predicting the probability of early mortality was generated and visualized as a nomogram. The performance of the prediction model was assessed by comparing the nomogram-predicted versus observed Kaplan-Meier estimates of survival probability.

Statistical analyses were performed using SPSS software (version 20.0) and $\mathrm{R}$ software (version 3.3.0). The following R packages were used: "rms" (Frank E Harrell), "plyr" (Hadley Wickham), "survival" (Terry M Therneau), "survminer" (Alboukadel Kassambara), "stargazer" (Marek Hlavac), "neuralnet" (Stefan Fritsch), "DynNom" (Amirhossein Jalali), "pROC" (Xavier Robin), "forestplot" (Max Gordon) (Thomas Lumley), and "regplot" (Roger Marshall). In two-sided tests, $P<0.05$ was considered statistically significant.

\section{Result}

\section{Patient characteristics}

The clinical characteristics of the cohort are shown in Table 1 . The average patient age was 40.09 years, with a range from 18 to 70 . A total of $69.8 \%$ of the patients were male. The tumor locations from most to least frequent were the frontal lobe for 38 (35.9\%), temporal lobe for $21(19.8 \%)$, multiple lobes for $28(26.4 \%)$ and other locations, including the parietal lobe, occipital lobe, cerebellum for 19 (17.9\%) patients. A total of 52.8\% had epilepsy as the initial symptom. The WHO grade at first diagnosis was II for $77(72.6 \%)$ and III for 29 patients (27.4\%). The pathology types at first diagnosis were astrocytoma for 62 (58.5\%) and oligodendroglioma for 44 patients (41.5\%). During follow-up, malignant transformation of the tumor was diagnosed for 33 (31.1\%) patients.

P53 mutation accounted for $77(72.6 \%)$ cases, $1 \mathrm{p} / 19 \mathrm{q}$ codeletion accounted for $47(44.3 \%)$ cases, IDH1 ${ }^{\mathrm{R} 132 \mathrm{H}}$ mutation accounted for $73(68.9 \%)$ cases, ATRX mutation accounted for $66(62.3 \%)$ cases, and the average Ki-67 index was 17.04 with a range from 0 to 80 . A total of $64.2 \%$ of the patients received Stupp standard radiochemotherapy after the first diagnosis. The average values of the laboratory test markers were as follows: NLR:
Table 1 Summary of Clinical Characteristics of LGG Patients Who Undergo Recurrence

\begin{tabular}{|c|c|}
\hline Characteristic & All Patients, $N=106$ \\
\hline \multicolumn{2}{|l|}{ Age, years } \\
\hline Average & 40.09 \\
\hline Range & $18-70$ \\
\hline \multicolumn{2}{|l|}{ Gender, n (\%) } \\
\hline Female & $32(30.2 \%)$ \\
\hline Male & $74(69.8 \%)$ \\
\hline \multicolumn{2}{|l|}{ Location, n (\%) } \\
\hline Frontal & $38(35.9 \%)$ \\
\hline Temporal & $21(19.8 \%)$ \\
\hline Multi & $28(26.4 \%)$ \\
\hline Other & $19(17.9 \%)$ \\
\hline \multicolumn{2}{|c|}{ Initial symptom, $\mathrm{n}(\%)$} \\
\hline Epilepsy & $56(52.8 \%)$ \\
\hline Other & $50(47.2 \%)$ \\
\hline \multicolumn{2}{|c|}{ First diagnosed grade, $\mathrm{n}(\%)$} \\
\hline WHO ॥ & $77(72.6 \%)$ \\
\hline WHO III & $29(27.4 \%)$ \\
\hline \multicolumn{2}{|c|}{ First diagnosed pathology type, n (\%) } \\
\hline Astrocytoma & $62(58.5 \%)$ \\
\hline Oligodendroglial & $44(41.5 \%)$ \\
\hline \multicolumn{2}{|c|}{ Malignant transform, n (\%) } \\
\hline Yes & $33(31.1 \%)$ \\
\hline No & $73(68.9 \%)$ \\
\hline \multicolumn{2}{|l|}{ P53, n (\%) } \\
\hline Mutant & $77(72.6 \%)$ \\
\hline Wide & $29(27.4 \%)$ \\
\hline \multicolumn{2}{|c|}{ 1p/19q Codeletion, $n$ (\%) } \\
\hline Yes & $47(44.3 \%)$ \\
\hline No & $59(55.7 \%)$ \\
\hline \multicolumn{2}{|l|}{ IDH1-R132H, n (\%) } \\
\hline Mutant & $73(68.9 \%)$ \\
\hline Wide & $33(31.1 \%)$ \\
\hline \multicolumn{2}{|l|}{ ATRX, n (\%) } \\
\hline Mutant & $66(62.3 \%)$ \\
\hline Wide & $40(37.7 \%)$ \\
\hline \multicolumn{2}{|l|}{ Ki-67, n } \\
\hline Average & 17.04 \\
\hline Range & $0-80$ \\
\hline \multicolumn{2}{|c|}{ Adjuvant therapy, n (\%) } \\
\hline Stupp & $68(64.2 \%)$ \\
\hline Other & $38(35.8 \%)$ \\
\hline \multicolumn{2}{|c|}{ Blood work, average (IQR) } \\
\hline NLR, & $2.820(0.667-21.298)$ \\
\hline$P L R$ & $128.653(41.026-578.723)$ \\
\hline LMR, & $4.191(0.181-21.667)$ \\
\hline RDW, \% & $13.65(12.0-23.6)$ \\
\hline $\mathrm{FIB}, \mathrm{g} / \mathrm{L}$ & $2.74(1.26-14.30)$ \\
\hline $\mathrm{DD}, \mathrm{mg} / \mathrm{L}$ & $0.160(0.005-1.080)$ \\
\hline TTR, days & \\
\hline
\end{tabular}


Table 1 (continued)

\begin{tabular}{ll}
\hline Characteristic & All Patients, $\mathbf{N}=\mathbf{1 0 6}$ \\
\hline Average & 1806.58 \\
Range & $177-6310$ \\
PRS, days & \\
Average & 768.86 \\
Range & $39-1981$ \\
OS, days & \\
$\quad$ Average & 2575.43 \\
Range & $263-8176$ \\
Survival Status, $\mathbf{n}$ (\%) & \\
Alive & $42(39.6 \%)$ \\
Dead & $64(60.4 \%)$ \\
\hline
\end{tabular}

IDH1 Isocitrate dehydrogenase 1, ATRX Alpha thalassemia/mental retardation syndrome $X$-linked, NLR the neutrophil-to-lymphocyte ratio, $P L R$ the platelet-tolymphocyte ratio, $L M R$ the lymphocyte-to-monocyte ratio, $R D W$ Red blood cell distribution width, FIB fibrinogen, DD D-dimer, IQR Interquartile range, TTR Time to recurrence, $P R S$ Post recurrence survival, OS Overall survival

2.820 (IQR 0.667-21.298), PLR: 128.653 (IQR 41.026578.723), LMR: 4.191 (IQR 0.181-21.667), RDW: 13.65 (IQR 12.0-23.6), FIB: 2.74 (IQR 1.26-14.30), DD: 0.160 (IQR 0.005-1.080).

During follow-up, the average OS after the first diagnosis was 2575.43 days (263-8176 days), the average TTR between the first diagnosis and tumor progression was 1806.58 days (177-6310 days), and the average PRS was 768.86 days (39-1981 days).

The cutoff values of the six hematological factors for OS are shown in Supplementary Table 1 (NLR-1.815, PLR100.749, LMR-3.029, RDW-13.4, FIB-2.80, DD-0.187). According to these values, the patients were classified into binary categories. Table 2 compares the preoperative risk factors for postoperative death within 1 year after LGG recurrence. Twenty-three patients (21.7\%) died within 1 year after reoperation. Early mortality was significantly associated with epilepsy as an initial symptom, 1p/19q codeletion, NLR, PLR, RDW, FIB and DD.

\section{Univariate and multivariate analyses}

The univariate and multivariate Cox regression results are visualized in Fig. 1A and Fig. 1B. Eight predictive variables were associated with a higher risk of early death within 1 year after reoperation in the univariate Cox analysis: older age $(P=0.042)$, higher DD $(P=0.007)$, higher RDW $(P=0.048)$, higher PLR $(P=0.014)$, higher NLR $(P=0.047), 1 \mathrm{p} 19 \mathrm{q}$ intact $(P=0.019), \quad$ IDH $1^{\mathrm{R} 132 \mathrm{H}}$ mutation $(\mathrm{P}=0.048)$, lower FIB $(P=0.018)$, lack of Stupp concurrent chemoradiotherapy $(P=0.041)$, and an initial symptom of epilepsy $(\mathrm{P}=0.047)$. In multivariate $\mathrm{Cox}$ analysis, age $(\mathrm{HR}=1.058, \quad 95 \%$ CI $1.014-1.103 ; \quad P=0.009), \quad$ DD
Table 2 Comparison of Preoperative Risk Factors for Postoperative Death Within 1 Year After Tumor Recurrence

\begin{tabular}{|c|c|c|c|}
\hline Characteristics & $\begin{array}{l}\text { Death Within } \\
1 \text { Year (23) }\end{array}$ & $\begin{array}{l}\text { Death After } \\
1 \text { Year (83) }\end{array}$ & P value \\
\hline \multicolumn{4}{|l|}{ Age, years } \\
\hline Average & 41.83 & 39.61 & \multirow[t]{2}{*}{0.618} \\
\hline Range & $24-68$ & $18-70$ & \\
\hline \multicolumn{4}{|l|}{ Gender, n (\%) } \\
\hline Female & $5(21.7 \%)$ & $27(32.5 \%)$ & \multirow[t]{2}{*}{0.233} \\
\hline Male & $18(78.2 \%)$ & $56(67.5 \%)$ & \\
\hline \multicolumn{4}{|l|}{ Location, n (\%) } \\
\hline Frontal & $8(34.8 \%)$ & $30(37.3 \%)$ & \multirow[t]{4}{*}{0.953} \\
\hline Temporal & $4(17.4 \%)$ & $17(20.5 \%)$ & \\
\hline Multi & $6(26.1 \%)$ & $22(26.5 \%)$ & \\
\hline Other & $5(21.7 \%)$ & $14(15.7 \%)$ & \\
\hline \multicolumn{4}{|c|}{ Initial symptom, n (\%) } \\
\hline Epilepsy & $17(73.9 \%)$ & $39(47.0 \%)$ & \multirow[t]{2}{*}{0.019} \\
\hline Other & $6(26.1 \%)$ & $44(53.0 \%)$ & \\
\hline \multicolumn{4}{|c|}{ First diagnosed grade, n (\%) } \\
\hline WHO ॥ & $16(69.6 \%)$ & $61(73.5 \%)$ & \multirow[t]{2}{*}{0.447} \\
\hline WHO III & $7(30.4 \%)$ & $22(26.5 \%)$ & \\
\hline \multicolumn{4}{|c|}{ First diagnosed pathology type, n (\%) } \\
\hline Astrocytoma & $16(69.6 \%)$ & $46(55.4 \%)$ & \multirow[t]{2}{*}{0.164} \\
\hline Oligodendroglial & $7(30.4 \%)$ & $37(44.6 \%)$ & \\
\hline \multicolumn{4}{|c|}{ Malignant transform, n (\%) } \\
\hline Yes & $8(34.8 \%)$ & $25(30.1 \%)$ & \multirow[t]{2}{*}{0.425} \\
\hline No & $15(65.2 \%)$ & $58(69.9 \%)$ & \\
\hline \multicolumn{4}{|l|}{ P53, n (\%) } \\
\hline Mutant & $19(82.6 \%)$ & $58(69.9 \%)$ & \multirow[t]{2}{*}{0.172} \\
\hline Wide & $4(17.4 \%)$ & $25(30.1 \%)$ & \\
\hline \multicolumn{4}{|c|}{ 1p/19q Codeletion, $n$ (\%) } \\
\hline yes & $6(26.1 \%)$ & $41(49.4 \%)$ & \multirow[t]{2}{*}{0.038} \\
\hline No & $17(73.9 \%)$ & $42(50.6 \%)$ & \\
\hline \multicolumn{4}{|l|}{ IDH1, n (\%) } \\
\hline Mutant & 15 (65.2\%) & $58(69.9 \%)$ & \multirow[t]{2}{*}{0.425} \\
\hline Wide & $8(34.8 \%)$ & $25(30.1 \%)$ & \\
\hline \multicolumn{4}{|l|}{ ATRX, n (\%) } \\
\hline Mutant & $15(65.2 \%)$ & $51(61.4 \%)$ & \multirow[t]{2}{*}{0.470} \\
\hline Wide & $8(34.8 \%)$ & $32(38.6 \%)$ & \\
\hline \multicolumn{4}{|l|}{ Ki-67, n } \\
\hline Average & 16.13 & 17.95 & 0.870 \\
\hline Range & $2-80$ & $0-70$ & \\
\hline Adjuvant therapy, & & & \\
\hline Stupp & $10(43.5 \%)$ & $58(69.9 \%)$ & 0.019 \\
\hline Other & $13(56.5 \%)$ & $25(30.1 \%)$ & \\
\hline NLR, n (\%) & & & \\
\hline$\leq 1.815$ & $4(17.4 \%)$ & $32(38.6 \%)$ & 0.046 \\
\hline$>1.815$ & $19(82.6 \%)$ & $51(61.4 \%)$ & \\
\hline PLR, n (\%) & & & \\
\hline$\leq 100.749$ & $4(17.4 \%)$ & 36 (43.4\%) & 0.018 \\
\hline$>100.749$ & 19 (82.6\%) & 47 (56.6\%) & \\
\hline
\end{tabular}


Table 2 (continued)

\begin{tabular}{|c|c|c|c|}
\hline Characteristics & $\begin{array}{l}\text { Death Within } \\
1 \text { Year (23) }\end{array}$ & $\begin{array}{l}\text { Death After } \\
1 \text { Year (83) }\end{array}$ & $P$ value \\
\hline \multicolumn{4}{|l|}{ LMR, n (\%) } \\
\hline$\leq 3.029$ & $6(26.1 \%)$ & $27(32.5 \%)$ & 0.375 \\
\hline$>3.029$ & $17(73.9 \%)$ & $56(67.5 \%)$ & \\
\hline \multicolumn{4}{|l|}{ RDW, n\% (\%) } \\
\hline$\leq 13.4$ & $11(47.8 \%)$ & $59(71.1 \%)$ & 0.035 \\
\hline$>13.4$ & $12(52.2 \%)$ & $24(28.9 \%)$ & \\
\hline \multicolumn{4}{|l|}{ Fib, g/L, n (\%) } \\
\hline$\leq 2.80$ & $10(43.5 \%)$ & $58(69.9 \%)$ & 0.019 \\
\hline$>2.80$ & $13(56.5 \%)$ & 25 (30.1\%) & \\
\hline \multicolumn{4}{|l|}{$\mathrm{DD}, \mathrm{mg} / \mathrm{L}, \mathrm{n}(\%)$} \\
\hline$\leq 0.187$ & $11(47.8 \%)$ & $61(73.5 \%)$ & 0.020 \\
\hline$>0.187$ & $12(52.2 \%)$ & $22(26.5 \%)$ & \\
\hline \multicolumn{4}{|l|}{ TTR, days } \\
\hline Average & 1777.48 & 1814.64 & 0.908 \\
\hline Range & $216-5425$ & $177-6310$ & \\
\hline
\end{tabular}

IDH1 isocitrate dehydrogenase 1, ATRX alpha thalassemia/mental retardation syndrome X-linked, NLR the neutrophil-to-lymphocyte ratio, $P L R$ the platelet-tolymphocyte ratio, $L M R$ the lymphocyte-to-monocyte ratio, $R D W$ red blood cell distribution width, FIB fibrinogen, DD D-dimer, IQR interquartile range, TTR time to recurrence, $O S$ overall survival

$(\mathrm{HR}=2.707, \quad 95 \% \quad \mathrm{CI}=1.001-7.321) \quad$ and $\quad 1 \mathrm{p} 19 \mathrm{q}$ $(\mathrm{HR}=0.265,95 \% \mathrm{CI} 0.098-0.718 ; \mathrm{P}=0.009)$ remained independently associated with early mortality after reoperation.

\section{Nomogram and validation}

Estimated probabilities for early mortality for surgically treated recurrent tumors were obtained by the sum of each variable score (Fig. 2). The calibration plot for the probability of survival for 5 years and 7 years after the first diagnosis showed optimal agreement between the prediction from the nomogram and the actual observation (Fig. 3A, Fig. 3B). An online calculator for the final nomogram is available at https://warrenwrl.shinyapps. io/RecurrenceGliomaEarlyM/.

\section{Survival analysis for independent markers}

For the independent associated factors according to multivariate Cox analysis, recurrent LGG patients without $1 \mathrm{p} 19 \mathrm{q}$ codeletion showed a significantly poorer prognosis than those with $1 \mathrm{p} 19 \mathrm{q}$ codeletion (Fig. 4A), and similarly, patients of older age showed a significantly poorer prognosis than younger patients (Fig. 4B). The results indicate that for recurrent LGG, patient characteristics, preoperative blood test and oncological information of the prior LGG may identify high-risk patients in clinical practice.

\section{Discussion}

Although recent research indicates that LGG patients could gain a survival benefit from repeat surgery after tumor recurrence, surgical treatment has not been widely accepted as a standard recurrent LGG treatment protocol due to an insufficient number of studies. Notably, newly developed salvage radiochemotherapy on latestage recurrent glioma has demonstrated the potential to prolong OS, expanding the therapy choices for recurrent LGG. Thus, in this study, we provided a tumoral and hematological marker-based nomogram to identify highrisk patients who may succumb to death within a year after reresection of recurrent LGG. Furthermore, this nomogram is implemented in free online software provided for easy use by clinicians worldwide.

During hospital stay, blood tests can contain sufficient significant hematological markers. These hematological markers from peripheral blood tests play a vital role in the prognosis of various malignant tumors [5-7], suggesting that the hemostatic components are interconnected with cancer biology in some ways [8]. In the present study, we included 6 hematological markers with 3 systemic inflammatory markers and 2 coagulation factors from 106 lower-grade glioma patients in this nomogram to assess preoperative risk factors for early death. The DD, RDW, PLR, NLR, and Fib levels were found to be related to early death in recurrent LGG.

As indicators of chronic inflammation, the PLR and NLR show potential to be representative of the characteristics of the tumor microenvironment, and chronic inflammation may foster tumor progression [9]. The NLR is a marker of the systemic inflammatory response and has been found to act as a factor for a poor prognosis in many malignancies, such as colon, bladder, and prostate cancers. In glioblastoma, an NLR $>4$ independently leads to a worse prognosis [10]. Further research quantified the prognostic value of PLR, NLR, and LMR based on the IDH mutation status in glioma and noted that a low NLR was associated with a better prognosis in the IDH-wild glioblastoma group, while PLR was predictive of survival in patients with primary glioblastoma and the IDH-wild GBM group [11]. However, the predictive value of the NLR for early death in the LGG cohort has not yet been reported. We believe our results indicate that the preoperative NLR level might represent a cancer-related systemic inflammatory response and may be a factor in predicting early mortality in surgically treated recurrent LGG.

Cancer development and aggressiveness mostly rely on neoangiogenesis and metastasis promoted by cancer cells [12]. Numerous studies have illustrated that coagulation activation is directly related to tumor progression, and mitigating coagulation activation can both prevent 

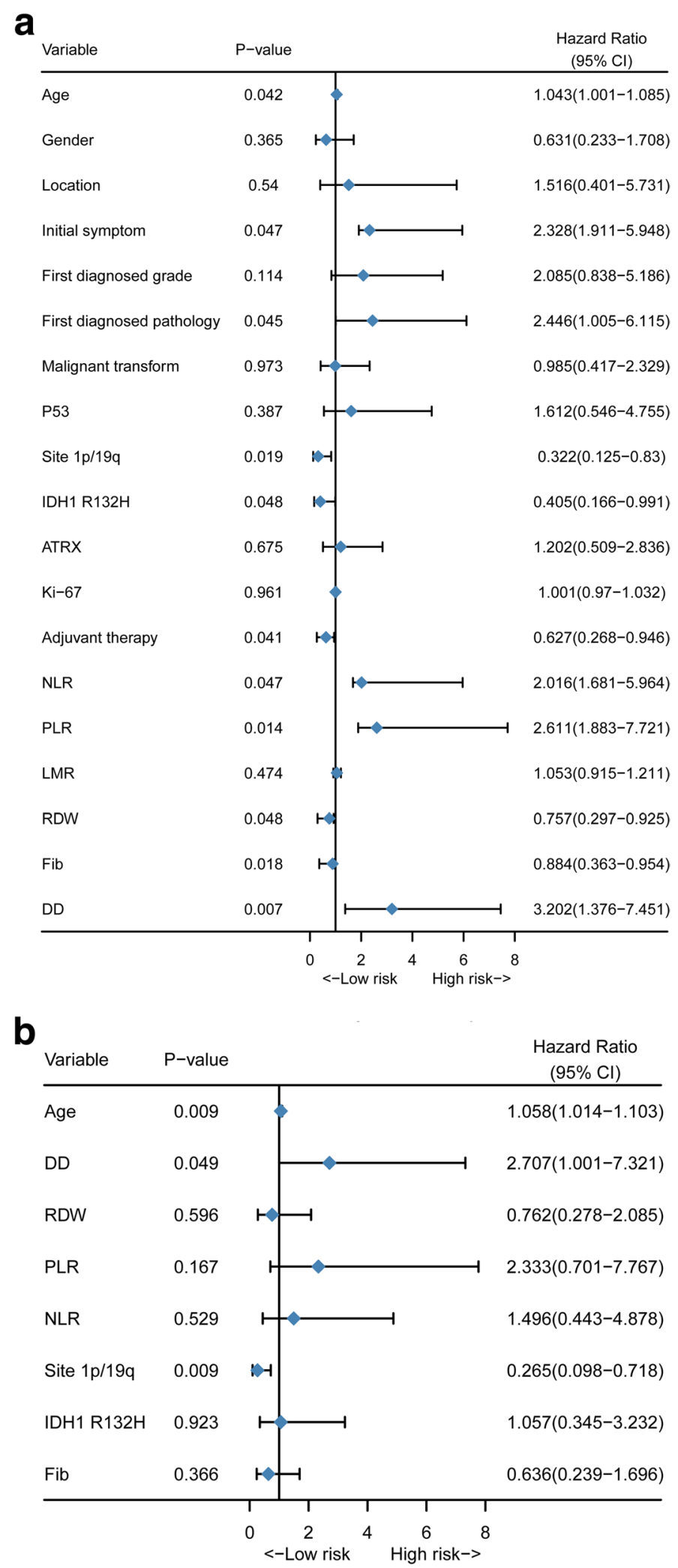

Fig. 1 Preoperative predictive factors for 1-year mortality in patients with surgically treated recurrent lower-grade glioma. (A) Forest plot of univariate Cox regression analysis of all clinical covariates; (B) Forest plot of multivariate Cox regression analysis of significant clinical covariates 


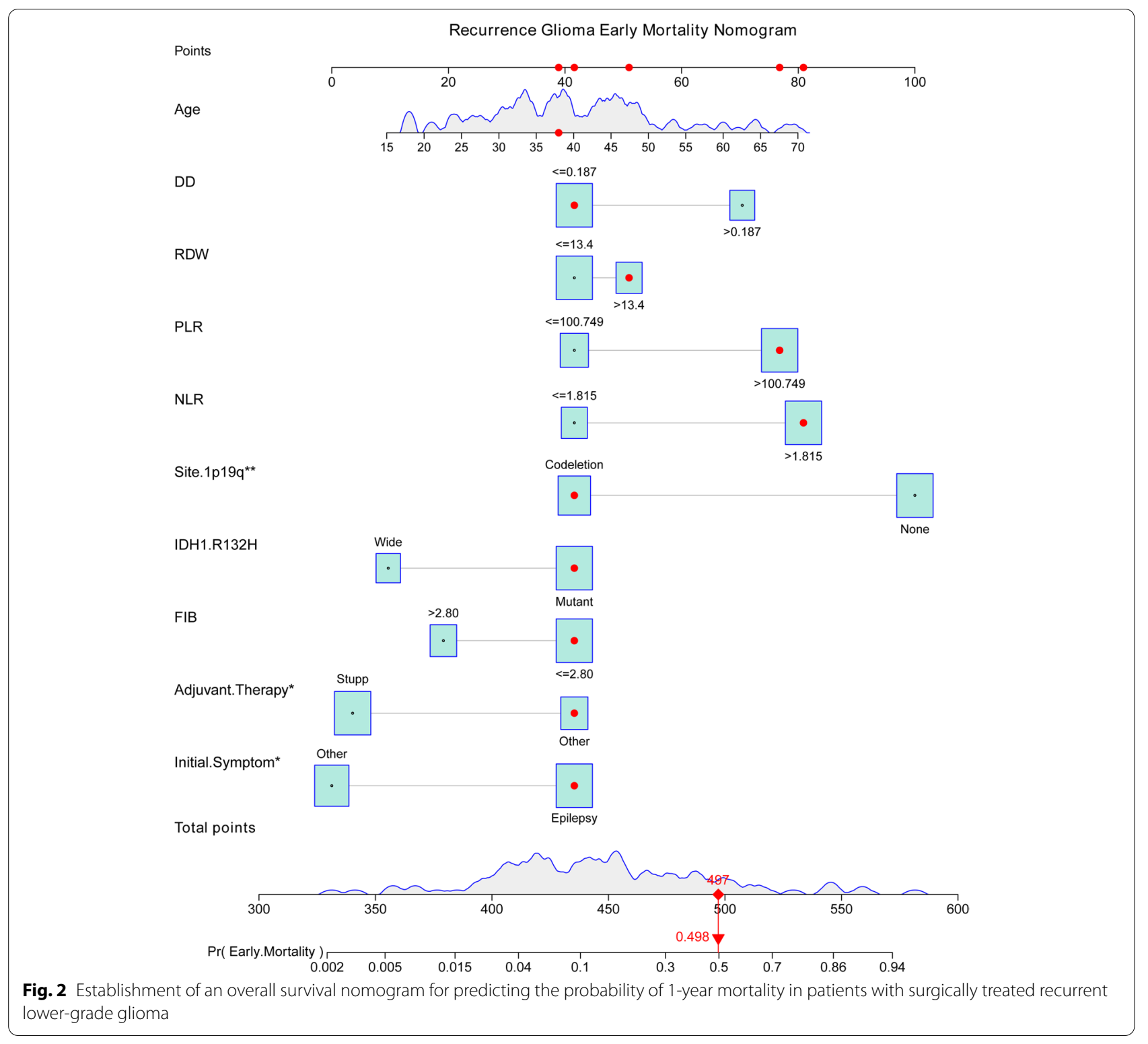

hemostatic complications and prolong survival in cancer patients [8]. An abnormal preoperative coagulation state not only indicates a poor general condition but also implies active tumor growth. As a crucial component in hemostasis and fibrinolysis activation, the $\mathrm{D}$-dimer level is often linked with a poor prognosis in several cancers of the body, such as lung, ovarian, gastric and liver cancers [13-15]. Our research indicates that an elevated preoperative plasma value of $\mathrm{D}$-dimer in patients with recurrent LGG is a risk factor for a higher incidence of death within a year after reoperation.

Under the 2016 WHO classification, 1p19q codeletion status is a necessary subtype that must be detected for WHO II-III astrocytoma with an IDH mutation or
WHO II-III oligodendroglioma. 1p19q codeletion represents the loss of heterozygosity in the short arm of chromosome $1(1 \mathrm{p})$ and the long arm of chromosome 19 (19q). The 1p19q state predicts the treatment response to chemotherapy, where codeletion predicted the effectiveness of temozolomide (TMZ) and procarbazine, lomustine, vincristine (PCV) chemotherapy and was associated with a better OS [16]. Recent studies have concluded that $1 \mathrm{p} 19 \mathrm{q}$ codeletion has independent significance on the overall survival of LGG patients who experience tumor recurrence and reoperation. A systematic analysis of clinical and biological significances of 1p19q genes indicates in $1 \mathrm{p} / 19 \mathrm{q}$ intact tumors, $1 \mathrm{p} 19 \mathrm{q}$-associated risk genes were enriched in processes that promote tumor growth 

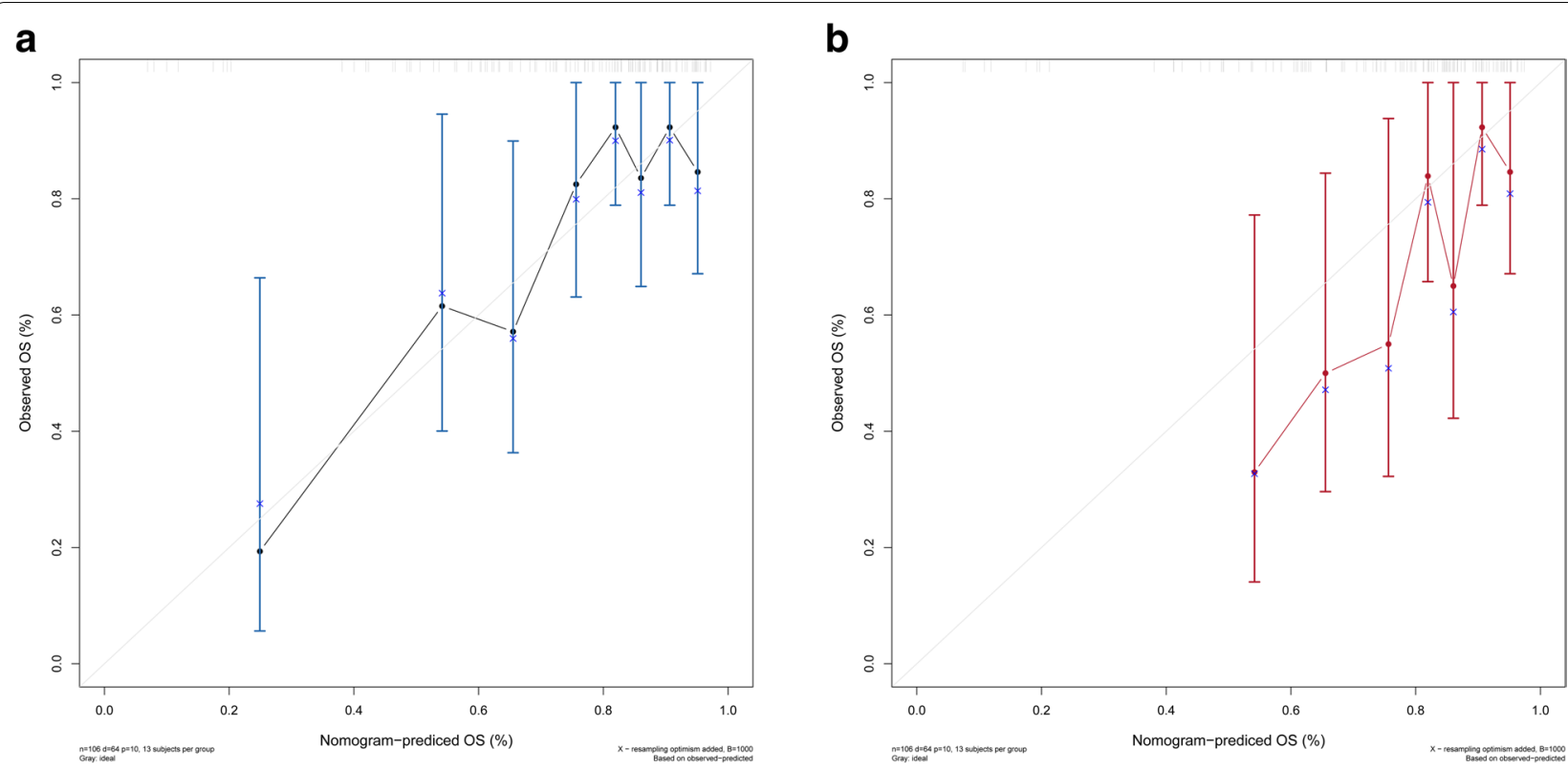

Fig. 3 Calibration curves for verifying the prediction accuracy of the nomogram for 5-year survival (A) and 7-year survival (B)

and migration, regulate tumor microenvironment and metabolism and promote drug resistance [17]. We further added the value of the influence of $1 \mathrm{p} 19 \mathrm{q}$ in a clinical cohort by demonstrating that patients with an intact $1 \mathrm{p} 19 \mathrm{q}$ site may experience earlier-than-expected mortality after reoperation of the recurrent tumor.

Mutations in the isocitrate dehydrogenase (IDH) 1 gene are commonly found in human glioma, with most lowergrade gliomas harboring a recurrent point mutation (IDH1 R132H). After the update of the 2016 WHO classification criteria, the importance of the IDH mutation was highlighted. Harboring the IDH1 mutation is linked to improved survival, and this holds true even in the setting of high-grade gliomas [18]. In this study, a conclusion of an insignificant association between IDH status and early mortality is reached, but we do not believe that this contradicts the findings of large-scale research. With LGG patients' 5 -year survival rates reaching $74 \%$ and some patients surviving more than 10 years after active clinical intervention, the survival advantage gained by the IDH mutation is often reflected over a longer period. The setting of 1-year time for early mortality in our study is a better reflection of reoperation-danger factors for the patient than the characteristic differences of the nature of the tumor.

Clinical presentation, which can be expressed as the initial presence of epilepsy, is a strong prognostic factor [19]. Epilepsy is the most common presenting symptom, observed in $80 \%$ of LGG patients [20]. We found that $52.8 \%$ of our cohort presented with epilepsy as the first symptom, which is in accordance with the findings with the TCGA dataset (53.2\%) and the OBTS dataset (61.2\%) [21]. A large cohort study reported that the presence of seizures at diagnosis was associated with more favorable outcomes, which might be due to the younger age of those whose initial symptoms was epilepsy. This appears contrary to our result, but the same study noted that a history of epileptic seizures at diagnosis was an independent predictor for malignant transformation: malignant-free survival was approximately 65 months and 40 months in LGG patients with and without a history of epileptic seizures at diagnosis, respectively [22]. Considering that a selective bias of inclusion of full recurrent LGG with an appreciable amount of malignant transformed glioma in our cohort, we believe that our result is robust.

One year mortality in reoperation of LGG patients is associated with various factors. Part of patients may succumb in a short while after the reresection. In view of our study result, we consider the adverse factors such as the abnormal systemic inflammatory and coagulation factors are reflecting the inferior state of the internal environment. The perioperative management and evaluation of glioma patients should balance the tumor characteristics and patients, in other words, treat the disease and the patients as while. Therefore, preoperative evaluation of patients' internal environment status and selecting appropriate therapy plan such as radical reoperation or salvage chemotherapy may contributes to improve oncologic outcome. 


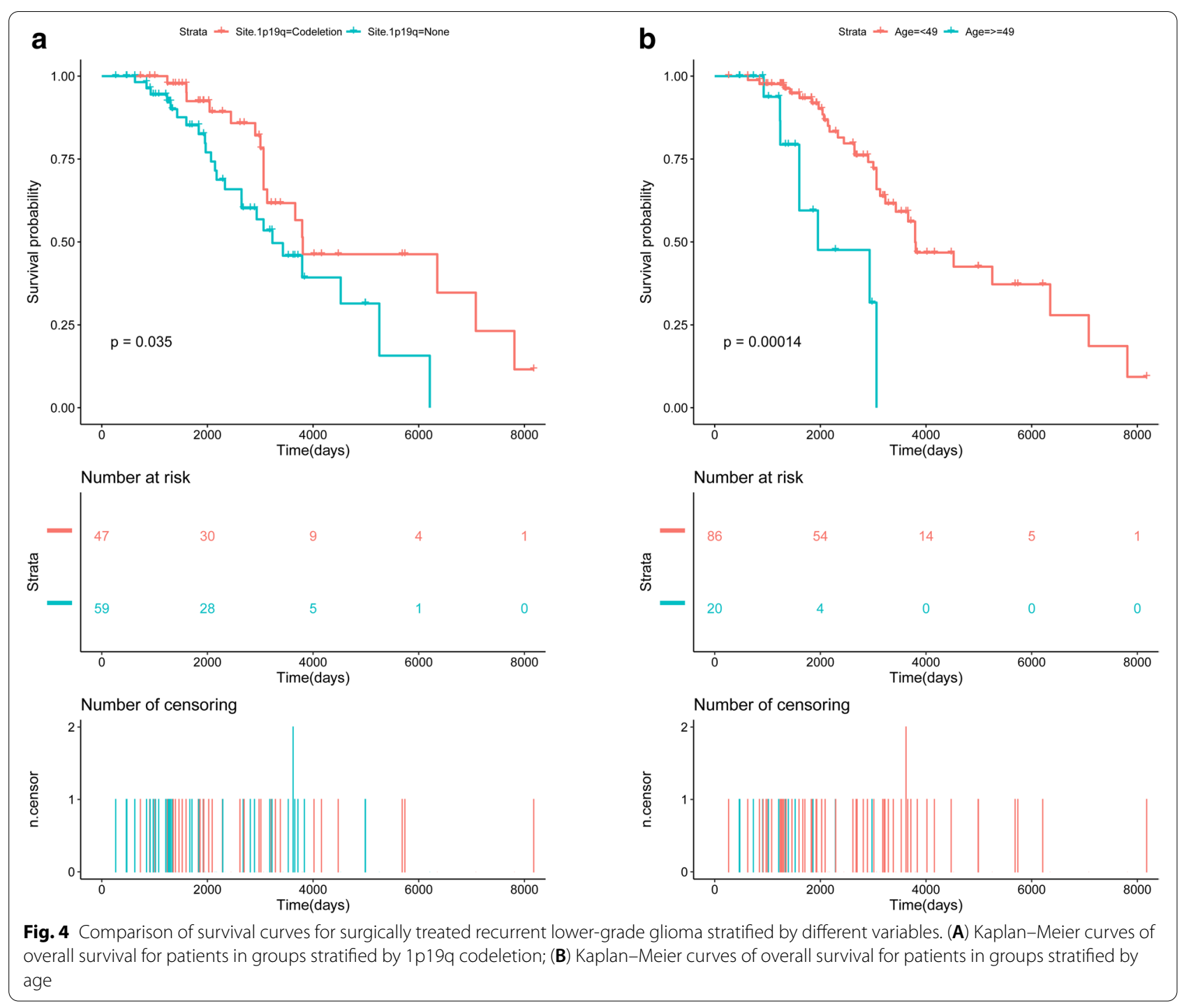

In all patients from our institution who underwent surgical resection of the primary tumor, a portion of patients were treated with a standard Stupp concurrent chemoradiotherapy plan, while others received TMZ, radiotherapy monotherapy, or close observation. The formulation of the adjuvant treatment plan is based on up-to-date glioma treatment guidance, and our study only illustrated the prediction of early mortality in patients with surgically treated, recurrent LGG based on these related factors. The enrolled patients in this study were identified at a single institution, and the prediction model may not be applicable to a multicenter cohort. As such, the result of the online calculator cannot be interpreted, as a nonsurgery plan should be preferred or used as a basis to change the treatment strategy for recurrent diffuse LGGs. Additionally, as we were limited by the wide time span of the longitudinal follow-up, the sample size was not sufficiently large, which may have led to bias in selection and analysis. Furthermore, due to funding and tissue limits, the study was limited to as a single-center cohort. Therefore, we disclosed all the initial data and original code, hoping to contribute to build a more precise and universal prediction model with a larger cohort and multicenter data.

\section{Conclusion}

In summary, we developed a nomogram to enable personalized estimation of 1-year mortality for patients with surgically treated recurrent lower-grade glioma based on patient clinical information, hematological markers and oncological factors. To facilitate the clinical use of this nomogram, free online software for its implementation is provided (https://warrenwrl.shinyapps.io/Recurrence GliomaEarlyM/). 


\begin{abstract}
Abbreviations
LGG: Lower-grade glioma; WHO: World Health Organization; CNS: Central Nervous System; sGBM: Secondary Glioblastoma; KPS: Karnofsky Performance Status; IDH1: Isocitrate dehydrogenase 1; ATRX: Alpha thalassemia/mental retardation syndrome X-linked; NLR: Neutrophil-to-Lymphocyte Ratio; PLR: Platelet-to-Lymphocyte Ratio; LMR: Lymphocyte-to-Monocyte Ratio; RDW: Red blood cell Distribution Width; FIB: Fibrinogen Level; DD: D-Dimer Level; OS: Overall Survival; PFS: Progression-Free Survival; RANO: Response Assessment in Neuro-Oncology; ROC: Receiver Operating Characteristic; AUC: Area Under the Curve; TMZ: Temozolomide; PCV: Procarbazine, lomustine, vincristine; TCGA: cancer genome atlas; OBTS: Ohio Brain Tumor Study.
\end{abstract}

\section{Supplementary Information}

The online version contains supplementary material available at https://doi. org/10.1186/s12885-022-09225-9.

Additional file 1 Supplementary Table S1. The optimal cutoff values of hematological factors for overall survival by Receiver operating characteristic (ROC) curve analysis.

\section{Acknowledgments}

Everyone who contributed to the article has been listed as an author.

\section{Declarations}

The Ethics Committee of the First Affiliated Hospital of Zhengzhou University approved this study, and the requirement for signed informed consent from patients was waived because of the retrospective nature of the analysis. All patient data were treated with confidentiality, in accordance with the Declaration of Helsinki.

\section{Authors' contributions}

Research design: RW, JL, XW. Data collection: RW. Sample collection and experiment conduction: RW, JL, FY and YX. Algorithm coding: RW and CZ. Statistical analysis: RW. Manuscript writing: RW. Manuscript submission: RW. All authors contributed to the article and approved the submitted version.

\section{Funding}

No funding was received for this research.

\section{Availability of data and materials}

The data that support the findings and the R-studio code that was generated in this study are openly available in Zenodo (DOl:https://doi.org/10.5281/ zenodo.5558404).

\section{Competing interests}

The authors report no conflicts of interest in this work.

\section{Author details}

'Department of Neurosurgery, The First Affiliated Hospital of Zhengzhou University, Jianshe East Road, Zhengzhou, China. ${ }^{2}$ Department of Neurosurgery, Sanbo Brain Hospital, Capital Medical University, Beijing, China. ${ }^{3}$ Department of Neurosurgery, West China Hospital, Sichuan University, Chengdu, China.

Received: 17 November 2021 Accepted: 24 January 2022

Published online: 28 January 2022 supratentorial low-grade glioma in adult patients. Acta Neurochir. 2009;151(11):1359-65.

4. Ramakrishna R, Hebb A, Barber J, Rostomily R, Silbergeld D. Outcomes in Reoperated low-grade gliomas. Neurosurgery. 2015;77(2):175-84 discussion 184 .

5. Charrier M, Mezquita L, Lueza B, Dupraz L, Planchard D, Remon J, et al. Circulating innate immune markers and outcomes in treatmentnaïve advanced non-small cell lung cancer patients. Eur J Cancer. 1990;2019(108):88-96.

6. Xu Z, Xu W, Cheng H, Shen W, Ying J, Cheng F, et al. The prognostic role of the platelet-lymphocytes ratio in gastric Cancer: a Meta-analysis. PLoS One. 2016;11(9):e0163719.

7. Li LQ, Bai ZH, Zhang LH, Zhang Y, Lu XC, Zhang Y, et al. Meta-analysis of hematological biomarkers as reliable indicators of soft tissue sarcoma prognosis. Front Oncol. 2020;10:30.

8. Falanga A, Marchetti M, Vignoli A. Coagulation and cancer: biological and clinical aspects. J Thromb Haemost. 2013;11(2):223-33.

9. Mantovani A, Allavena P, Sica A, Balkwill F. Cancer-related inflammation. Nature. 2008;454(7203):436-44.

10. Bambury RM, Teo MY, Power DG, Yusuf A, Murray S, Battley JE, et al. The association of pre-treatment neutrophil to lymphocyte ratio with overall survival in patients with glioblastoma multiforme. J Neuro-Oncol. 2013;114(1):149-54.

11. Wang PF, Song HW, Cai HQ, Kong LW, Yao K, Jiang T, et al. Preoperative inflammation markers and IDH mutation status predict glioblastoma patient survival. Oncotarget. 2017;8(30):50117-23.

12. Falanga A, Panova-Noeva M, Russo L. Procoagulant mechanisms in tumour cells. Best Pract Res Clin Haematol. 2009;22(1):49-60.

13. Gadducci A, Cosio S, Tana R, Genazzani AR. Serum and tissue biomarkers as predictive and prognostic variables in epithelial ovarian cancer. Crit Rev Oncol Hematol. 2009:69(1):12-27.

14. Tas F, Kilic L, Bilgin E, Keskin S, Sen F, Ciftci R, et al. Clinical and prognostic significance of coagulation assays in advanced epithelial ovarian cancer. Int J Gynecol Cancer. 2013;23(2):276-81.

15. Inal $T$, Anar C, Polat G, Ünsal I, Halilçolar H. The prognostic value of D-dimer in lung cancer. Clin Respir J. 2015;9(3):305-13.

16. McNamara MG, Jiang H, Lim-Fat MJ, Sahebjam S, Kiehl TR, Karamchandani J, et al. Treatment outcomes in 1p19q co-deleted/partially deleted gliomas. Can J Neurol Sci. 2017;44(3):288-94.

17. Chai RC, Zhang KN, Chang YZ, Wu F, Liu YQ, Zhao Z, et al. Systematically characterize the clinical and biological significances of 1 p 19q genes in 1p/19q non-codeletion glioma. Carcinogenesis. 2019;40(10):1229-39.

18. Karpel-Massler G, Nguyen TTT, Shang E, Siegelin MD. Novel IDH1-targeted glioma therapies. CNS drugs. 2019;33(12):1155-66.

19. Cavaliere R, Lopes MB, Schiff D. Low-grade gliomas: an update on pathology and therapy. Lancet Neurol. 2005;4(11):760-70.

20. Morshed RA, Young JS, Hervey-Jumper SL, Berger MS. The management of low-grade gliomas in adults. J Neurosurg Sci. 2019;63(4):450-7.

21. Gittleman H, Sloan AE, Barnholtz-Sloan JS. An independently validated survival nomogram for lower-grade glioma. Neuro-oncology. 2020;22(5):665-74

22. Pallud J, Audureau E, Blonski M, Sanai N, Bauchet L, Fontaine D, et al. Epileptic seizures in diffuse low-grade gliomas in adults. Brain. 2014;137(Pt 2):449-62.

\section{Publisher's Note}

Springer Nature remains neutral with regard to jurisdictional claims in published maps and institutional affiliations.

\section{References}

1. Wirsching H-G, Galanis E, Weller M: Glioblastoma Handbook of clinical neurology 2016, 134:381-397.

2. Brat DJ, Verhaak RG, Aldape KD, Yung WK, Salama SR, Cooper LA, et al. Comprehensive, integrative genomic analysis of diffuse lower-grade gliomas. N Engl J Med. 2015;372(26):2481-98.

3. Ahmadi R, Dictus C, Hartmann C, Zürn O, Edler L, Hartmann $\mathrm{M}$, et al. Long-term outcome and survival of surgically treated 